Eur. J. Math. Anal. 1 (2021) 133-150

doi: 10.28924/ada/ma.1.133

\title{
Lie Group Analysis of a Nonlinear Coupled System of Korteweg-de Vries Equations
}

\author{
Joseph Owuor Owino*, Benard Okelo \\ Department of Pure and Applied Mathematics, Jaramogi Oginga Odinga University of Science and \\ Technology, Box 210-40601, Bondo, Kenya \\ bnyaare@yahoo.com, josephowuorowino@gmail.com \\ *Correspondence: josephowuorowino@gmail.com
}

\begin{abstract}
AвStRaCt. In this paper, we consider coupled Korteweg-de Vries equations that model the propagation of shallow water waves, ion-acoustic waves in plasmas, solitons, and nonlinear perturbations along internal surfaces between layers of different densities in stratified fluids, for example propagation of solitons of long internal waves in oceans. The method of Lie group analysis is used to on the system to obtain symmetry reductions. Soliton solutions are constructed by use of a linear combination of time and space translation symmetries. Furthermore, we compute conservation laws in two ways that is by multiplier method and by an application of new conservation theorem developed by Nail Ibragimov.
\end{abstract}

\section{INTRODUCTION}

The dynamics of shallow-water waves, ion-acoustic waves in plasmas, and long internal waves in oceans can be described by coupled $\mathrm{KdV}$ equations. The equations are derived from the classical $k d V$ equation. This section extends the previous study of $k d V$ equations to that of a coupled nonlinear system. From the Kortweg-de Vries equation

$$
q_{t}+\alpha q q_{x}+\beta q_{x x x}=0
$$

for $\alpha$ and $\beta$ as constants, we let

$$
q(t, x)=u(t, x)+i v(t, x)
$$

where $i^{2}=-1$. Then substituting (2) into (1) and separating the real and imaginary parts, we obtain

$$
\Delta_{1} \equiv u_{t}+\alpha u u_{x}-\alpha v v_{x}+\beta u_{x x x}=0, \quad \Delta_{2} \equiv v_{t}+\alpha u v_{x}+\alpha v u_{x}+\beta v_{x x x}=0,
$$

Received: 3 Sep 2021.

Key words and phrases. coupled KdV equations; Lie group analysis; group-invariant solutions; stationary solutions; symmetry reductions; soliton; multipliers; conservation laws. 
which is a nonlinear system of coupled KdV equations. We perform Lie symmetry analysis on (3), that is, we obtain Lie point symmetries, invariant solutions and conservation laws of (3).This paper uses symmetry analysis method to construct exact solutions and conservation laws for a nonlinear coupled kdV system (3).

\section{Preliminaries}

In this section, we outline preliminary concepts which are useful in the sequel. In Euclidean spaces $\mathbb{R}^{n}$ of $x=x^{i}$ independent variables and $\mathbb{R}^{m}$ of $u=u^{\alpha}$ dependent variables, we consider the transformations

$$
T_{\epsilon}: \quad \bar{x}^{i}=\varphi^{i}\left(x^{i}, u^{\alpha}, \epsilon\right), \quad \bar{u}^{\alpha}=\psi^{\alpha}\left(x^{i}, u^{\alpha}, \epsilon\right),
$$

involving the continuous parameter $\epsilon$ which ranges from a neighbourhood $\mathcal{N}^{\prime} \subset \mathcal{N} \subset \mathbb{R}$ of $\epsilon=0$ where the functions $\varphi^{i}$ and $\psi^{\alpha}$ differentiable and analytic in the parameter $\epsilon$.

Definition 2.1. The set $\mathcal{G}$ of transformations given by (4) is a local Lie group if it holds true that

(1) (i). (Closure) Given $T_{\epsilon_{1}}, T_{\epsilon_{2}} \in \mathcal{G}$, for $\epsilon_{1}, \epsilon_{2} \in \mathcal{N}^{\prime} \subset \mathcal{N}$, then $T_{\epsilon_{1}} T_{\epsilon_{2}}=T_{\epsilon_{3}} \in \mathcal{G}, \epsilon_{3}=$ $\phi\left(\epsilon_{1}, \epsilon_{2}\right) \in \mathcal{N}$.

(2) (ii). (Identity) There exists a unique $T_{0} \in \mathcal{G}$ if and only if $\epsilon=0$ such that $T_{\epsilon} T_{0}=T_{0} T_{\epsilon}=T_{\epsilon}$.

(3) (iii). (Inverse) There exists a unique $T_{\epsilon^{-1}} \in \mathcal{G}$ for every transformation $T_{\epsilon} \in \mathcal{G}$, where $\epsilon \in \mathcal{N}^{\prime} \subset \mathcal{N}$ and $\epsilon^{-1} \in \mathcal{N}$ such that $T_{\epsilon} T_{\epsilon^{-1}}=T_{\epsilon^{-1}} T_{\epsilon}=T_{0}$.

Remark 2.2. Associativity of the group $\mathcal{G}$ in (4) follows from (1).

In the system,

$$
\Delta_{\alpha}\left(x^{i}, u^{\alpha}, u_{(1)}, \ldots, u_{(\pi)}\right)=\Delta_{\alpha}=0,
$$

the variables $u^{\alpha}$ are dependent. The partial derivatives $u_{(1)}=\left\{u_{i}^{\alpha}\right\}, u_{(2)}=\left\{u_{i j}^{\alpha}\right\}, \ldots, u_{(\pi)}=$ $\left\{u_{i_{1} \ldots i_{\pi}}^{\alpha}\right\}$, are of the first, second, ..., up to the $\pi$ th-orders.

Denoting

$$
D_{i}=\frac{\partial}{\partial x^{i}}+u_{i}^{\alpha} \frac{\partial}{\partial u^{\alpha}}+u_{i j}^{\alpha} \frac{\partial}{\partial u_{j}^{\alpha}}+\ldots
$$

the total differentiation operator with respect to the variables $x^{i}$ and $\delta_{i}^{j}$, the Kronecker delta, we have

$$
D_{i}\left(x^{j}\right)=\delta_{i}^{j},{ }^{\prime}, u_{i}^{\alpha}=D_{i}\left(u^{\alpha}\right), \quad u_{i j}^{\alpha}=D_{j}\left(D_{i}\left(u^{\alpha}\right)\right), \ldots,
$$

where $u_{i}^{\alpha}$ defined in (7) are differential variables [7].

Consider the local Lie group $\mathcal{G}$ given by the transformations

$$
\bar{x}^{i}=\varphi^{i}\left(x^{i}, u^{\alpha}, \epsilon\right),\left.\quad \varphi^{i}\right|_{\epsilon=0}=x^{i}, \quad \bar{u}^{\alpha}=\psi^{\alpha}\left(x^{i}, u^{\alpha}, \epsilon\right),\left.\quad \psi^{\alpha}\right|_{\epsilon=0}=u^{\alpha},
$$

where the symbol $\left.\right|_{\epsilon=0}$ means evaluated on $\epsilon=0$. 
Definition 2.3. The construction of the group $\mathcal{G}$ given by (8) is an equivalence of the computation of infinitesimal transformations

$$
\bar{x}^{i} \approx x^{i}+\xi^{i}\left(x^{i}, u^{\alpha}\right) \epsilon,\left.\quad \varphi^{i}\right|_{\epsilon=0}=x^{i}, \quad \bar{u}^{\alpha} \approx u^{\alpha}+\eta^{\alpha}\left(x^{i}, u^{\alpha}\right) \epsilon,\left.\quad \psi^{\alpha}\right|_{\epsilon=0}=u^{\alpha},
$$

obtained from (4) by a Taylor series expansion of $\varphi^{i}\left(x^{i}, u^{\alpha}, \epsilon\right)$ and $\psi^{i}\left(x^{i}, u^{\alpha}, \epsilon\right)$ in $\epsilon$ about $\epsilon=0$ and keeping only the terms linear in $\epsilon$, where

$$
\xi^{i}\left(x^{i}, u^{\alpha}\right)=\left.\frac{\partial \varphi^{i}\left(x^{i}, u^{\alpha}, \epsilon\right)}{\partial \epsilon}\right|_{\epsilon=0}, \quad \eta^{\alpha}\left(x^{i}, u^{\alpha}\right)=\left.\frac{\partial \psi^{\alpha}\left(x^{i}, u^{\alpha}, \epsilon\right)}{\partial \epsilon}\right|_{\epsilon=0} .
$$

Remark 2.4. The symbol of infinitesimal transformations, $X$, is used to write (9) as

$$
\bar{x}^{i} \approx(1+X) x^{i}, \quad \bar{u}^{\alpha} \approx(1+X) u^{\alpha},
$$

where

$$
x=\xi^{i}\left(x^{i}, u^{\alpha}\right) \frac{\partial}{\partial x^{i}}+\eta^{\alpha}\left(x^{i}, u^{\alpha}\right) \frac{\partial}{\partial u^{\alpha}},
$$

is the generator of the group $\mathcal{G}$ given by (8).

Remark 2.5. To obtain transformed derivatives from (4), we use a change of variable formulae

$$
D_{i}=D_{i}\left(\varphi^{j}\right) \bar{D}_{j}
$$

where $\bar{D}_{j}$ is the total differentiation in the variables $\bar{x}^{i}$. This means that

$$
\bar{u}_{i}^{\alpha}=\bar{D}_{i}\left(\bar{u}^{\alpha}\right), \bar{u}_{i j}^{\alpha}=\bar{D}_{j}\left(\bar{u}_{i}^{\alpha}\right)=\bar{D}_{i}\left(\bar{u}_{j}^{\alpha}\right) .
$$

If we apply the change of variable formula given in (13) on $\mathcal{G}$ given by (8), we get

$$
D_{i}\left(\psi^{\alpha}\right)=D_{i}\left(\varphi^{j}\right), \bar{D}_{j}\left(\bar{u}^{\alpha}\right)=\bar{u}_{j}^{\alpha} D_{i}\left(\varphi^{j}\right) .
$$

Expansion of (15) yields

$$
\left(\frac{\partial \varphi^{j}}{\partial x^{i}}+u_{i}^{\beta} \frac{\partial \varphi^{j}}{\partial u^{\beta}}\right) \bar{u}_{j}^{\beta}=\frac{\partial \psi^{\alpha}}{\partial x^{i}}+u_{i}^{\beta} \frac{\partial \psi^{\alpha}}{\partial u^{\beta}} .
$$

The variables $\bar{u}_{i}^{\alpha}$ can be written as functions of $x^{i}, u^{\alpha}, u_{(1)}$, that is

$$
\bar{u}_{i}^{\alpha}=\Phi^{\alpha}\left(x^{i}, u^{\alpha}, u_{(1)}, \epsilon\right),\left.\quad \Phi^{\alpha}\right|_{\epsilon=0}=u_{i}^{\alpha} .
$$

Definition 2.6. The transformations in the space of the variables $x^{i}, u^{\alpha}, u_{(1)}$ given in (8) and (17) form the first prolongation group $\mathcal{G}^{[1]}$.

Definition 2.7. Infinitesimal transformation of the first derivatives is

$$
\bar{u}_{i}^{\alpha} \approx u_{i}^{\alpha}+\zeta_{i}^{\alpha} \epsilon, \quad \text { where } \quad \zeta_{i}^{\alpha}=\zeta_{i}^{\alpha}\left(x^{i}, u^{\alpha}, u_{(1)}, \epsilon\right)
$$

Remark 2.8. In terms of infinitesimal transformations, the first prolongation group $\mathcal{G}^{[1]}$ is given by (9) and (18). 
Definition 2.9. By using the relation given in (15) on the first prolongation group $\mathcal{G}^{[1]}$ given by Definition 2.6, we obtain [5]

$$
D_{i}\left(x^{j}+\xi^{j} \epsilon\right)\left(u_{j}^{\alpha}+\zeta_{j}^{\alpha} \epsilon\right)=D_{i}\left(u^{\alpha}+\eta^{\alpha} \epsilon\right), \quad \text { which gives } u_{i}^{\alpha}+\zeta_{j}^{\alpha} \epsilon+u_{j}^{\alpha} \epsilon D_{i} \xi^{j}=u_{i}^{\alpha}+D_{i} \eta^{\alpha} \epsilon
$$

and thus

$$
\zeta_{i}^{\alpha}=D_{i}\left(\eta^{\alpha}\right)-u_{j}^{\alpha} D_{i}\left(\xi^{j}\right)
$$

is the first prolongation formula.

Remark 2.10. Similarly, we get higher order prolongations [8],

$$
\zeta_{i j}^{\alpha}=D_{j}\left(\zeta_{i}^{\alpha}\right)-u_{i \kappa}^{\alpha} D_{j}\left(\xi^{\kappa}\right), \quad \ldots, \quad \zeta_{i_{1}, \ldots, i_{\kappa}}^{\alpha}=D_{i_{\kappa}}\left(\zeta_{i_{1}, \ldots, i_{\kappa-1}}^{\alpha}\right)-u_{i_{1}, i_{2}, \ldots, i_{\kappa-1} j}^{\alpha} D_{i_{\kappa}}\left(\xi^{j}\right) .
$$

Remark 2.11. The prolonged generators of the prolongations $\mathcal{G}^{[1]}, \ldots, \mathcal{G}^{[\kappa]}$ of the group $\mathcal{G}$ are

$$
X^{[1]}=X+\zeta_{i}^{\alpha} \frac{\partial}{\partial u_{i}^{\alpha}}, \ldots, X^{[\kappa]}=X^{[\kappa-1]}+\zeta_{i_{1}, \ldots, i_{\kappa}}^{\alpha} \frac{\partial}{\partial \zeta_{i_{1}, \ldots, i_{\kappa}}^{\alpha}}, \kappa \geq 1,
$$

where $X$ is the group generator given by (12).

Definition 2.12. A function $\Gamma\left(x^{i}, u^{\alpha}\right)$ is called an invariant of the group $\mathcal{G}$ of transformations given by (4) if

$$
\Gamma\left(\bar{x}^{i}, \bar{u}^{\alpha}\right)=\Gamma\left(x^{i}, u^{\alpha}\right) .
$$

Theorem 2.13. A function $\Gamma\left(x^{i}, u^{\alpha}\right)$ is an invariant of the group $\mathcal{G}$ given by (4) if and only if it solves the following first-order linear PDE: [5]

$$
X \Gamma=\xi^{i}\left(x^{i}, u^{\alpha}\right) \frac{\partial \Gamma}{\partial x^{i}}+\eta^{\alpha}\left(x^{i}, u^{\alpha}\right) \frac{\partial \Gamma}{\partial u^{\alpha}}=0 .
$$

From Theorem (2.13), we have the following result.

Theorem 2.14. The local Lie group $\mathcal{G}$ of transformations in $\mathbb{R}^{n}$ given by (4) [7] has precisely $n-1$ functionally independent invariants. One can take, as the basic invariants, the left-hand sides of the first integrals

$$
\psi_{1}\left(x^{i}, u^{\alpha}\right)=c_{1}, \ldots, \psi_{n-1}\left(x^{i}, u^{\alpha}\right)=c_{n-1}
$$

of the characteristic equations for (24):

$$
\frac{\mathrm{d} x^{i}}{\xi^{i}\left(x^{i}, u^{\alpha}\right)}=\frac{\mathrm{d} u^{\alpha}}{\eta^{\alpha}\left(x^{i}, u^{\alpha}\right)} .
$$


Definition 2.15. The vector field $X(12)$ is a Lie point symmetry of the PDE system (5) if the determining equations

$$
\left.X^{[\pi]} \Delta_{\alpha}\right|_{\Delta_{\alpha}=0}=0, \quad \alpha=1, \ldots, m, \quad \pi \geq 1
$$

are satisfied, where $\left.\right|_{\Delta_{\alpha}=0}$ means evaluated on $\Delta_{\alpha}=0$ and $X^{[\pi]}$ is the $\pi$-th prolongation of $X$.

Definition 2.16. The Lie group $\mathcal{G}$ is a symmetry group of the PDE system given in (5) if the PDE system (5) is form-invariant, that is

$$
\Delta_{\alpha}\left(\bar{x}^{i}, \bar{u}^{\alpha}, \bar{u}_{(1)}, \ldots, \bar{u}_{(\pi)}\right)=0
$$

Theorem 2.17. Given the infinitesimal transformations in (8), the Lie group $\mathcal{G}$ in (4) is found by integrating the Lie equations

$$
\frac{\mathrm{d} \bar{x}^{i}}{\mathrm{~d} \epsilon}=\xi^{i}\left(\bar{x}^{i}, \bar{u}^{\alpha}\right),\left.\quad \bar{x}^{i}\right|_{\epsilon=0}=x^{i}, \quad \frac{\mathrm{d} \bar{u}^{\alpha}}{\mathrm{d} \epsilon}=\eta^{\alpha}\left(\bar{x}^{i}, \bar{u}^{\alpha}\right),\left.\quad \bar{u}^{\alpha}\right|_{\epsilon=0}=u^{\alpha} .
$$

Definition 2.18. A vector space $\mathcal{V}_{r}$ of operators [5] $X(12)$ is a Lie algebra if for any two operators, $X_{i}, X_{j} \in \mathcal{V}_{r}$, their commutator

$$
\left[X_{i}, X_{j}\right]=X_{i} X_{j}-X_{j} X_{i}
$$

is in $\mathcal{V}_{r}$ for all $i, j=1, \ldots, r$.

Remark 2.19. The commutator satisfies the properties of bilinearity, skew symmetry and the Jacobi identity [5].

Theorem 2.20. The set of solutions of the determining equation given by (27) forms a Lie algebra [5].

The methods of ( $\left.G^{\prime} / G\right)$-expansion method [20], Extended Jacobi elliptic function expansion [21] and Kudryashov [22] are usually applied after symmetry reductions. Let a system of $\pi$ th-order PDEs be given by (5).

Definition 2.21. The Euler-Lagrange operator $\delta / \delta u^{\alpha}$ is

$$
\frac{\delta}{\delta u^{\alpha}}=\frac{\partial}{\partial u^{\alpha}}+\sum_{\kappa \geq 1}(-1)^{\kappa} D_{i_{1}}, \ldots, D_{i_{\kappa}} \frac{\partial}{\partial u_{i_{1} i_{2} \ldots i_{\kappa}}^{\alpha}},
$$

and the Lie- Bäcklund operator in abbreviated form [5] is

$$
X=\xi^{i} \frac{\partial}{\partial x^{i}}+\eta^{\alpha} \frac{\partial}{\partial u^{\alpha}}+\ldots
$$

Remark 2.22. The Lie- Bäcklund operator (32) in its prolonged form is

$$
X=\xi^{i} \frac{\partial}{\partial x^{i}}+\eta^{\alpha} \frac{\partial}{\partial u^{\alpha}}+\sum_{\kappa \geq 1} \zeta_{i_{1} \ldots i_{\kappa}} \frac{\partial}{\partial u_{i_{1} i_{2} \ldots i_{\kappa}}^{\alpha}},
$$


where

$$
\zeta_{i}^{\alpha}=D_{i}\left(W^{\alpha}\right)+\xi^{j} u_{i j}^{\alpha}, \quad \ldots, \zeta_{i_{1} \ldots i_{\kappa}}^{\alpha}=D_{i_{1} \ldots i_{\kappa}}\left(W^{\alpha}\right)+\xi^{j} u_{j i_{1} \ldots i_{\kappa}}^{\alpha}, \quad j=1, \ldots, n
$$

and the Lie characteristic function is

$$
W^{\alpha}=\eta^{\alpha}-\xi^{j} u_{j}^{\alpha}
$$

Remark 2.23. The characteristic form of Lie- Bäcklund operator (33) is

$$
X=\xi^{i} D_{i}+W^{\alpha} \frac{\partial}{\partial u^{\alpha}}+D_{i_{1} \ldots i_{\kappa}}\left(W^{\alpha}\right) \frac{\partial}{\partial u_{i_{1} i_{2} \ldots i_{\kappa}}^{\alpha}} .
$$

Remark 2.24. Noether's Theorem is applicable to systems from variational problems

Definition 2.25. A function $\wedge^{\alpha}\left(x^{i}, u^{\alpha}, u_{(1)}, \ldots\right)=\wedge^{\alpha}$, is a multiplier of the PDE system given by (5) if it satisfies the condition that [16]

$$
\wedge^{\alpha} \Delta_{\alpha}=D_{i} T^{i}
$$

where $D_{i} T^{i}$ is a divergence expression.

Definition 2.26. To find the multipliers $\wedge^{\alpha}$, one solves the determining equations (38) [3],

$$
\frac{\delta}{\delta u^{\alpha}}\left(\wedge^{\alpha} \Delta_{\alpha}\right)=0
$$

The technique [9] enables one to construct conserved vectors associated with each Lie point symmetry of the PDE system given by (5).

Definition 2.27. The adjoint equations of the system given by (5) are

$$
\Delta_{\alpha}^{*}\left(x^{i}, u^{\alpha}, v^{\alpha}, \ldots, u_{(\pi)}, v_{(\pi)}\right) \equiv \frac{\delta}{\delta u^{\alpha}}\left(v^{\beta} \Delta_{\beta}\right)=0
$$

where $v^{\alpha}$ is the new dependent variable.

Definition 2.28. Formal Lagrangian $\mathcal{L}$ of the system (5) and its adjoint equations (39) is [9]

$$
\mathcal{L}=v^{\alpha} \Delta_{\alpha}\left(x^{i}, u^{\alpha}, u_{(1)}, \ldots, u_{(\pi)}\right)
$$

Theorem 2.29. Every infinitesimal symmetry Xof the system given by (5) leads to conservation laws [9]

$$
\left.D_{i} T^{i}\right|_{\Delta_{\alpha=0}}=0
$$

where the conserved vector

$$
\begin{gathered}
T^{i}=\xi^{i} \mathcal{L}+W^{\alpha}\left[\frac{\partial \mathcal{L}}{\partial u_{i}^{\alpha}}-D_{j}\left(\frac{\partial \mathcal{L}}{\partial u_{i j}^{\alpha}}\right)+D_{j} D_{k}\left(\frac{\partial \mathcal{L}}{\partial u_{i j k}^{\alpha}}\right)-\ldots\right]+ \\
D_{j}\left(W^{\alpha}\right)\left[\frac{\partial \mathcal{L}}{\partial u_{i j}^{\alpha}}-D_{k}\left(\frac{\partial \mathcal{L}}{\partial u_{i j k}^{\alpha}}\right)+\ldots\right]+D_{j} D_{k}\left(W^{\alpha}\right)\left[\frac{\partial \mathcal{L}}{\partial u_{i j k}^{\alpha}}-\ldots\right] .
\end{gathered}
$$




\section{MAIN RESULTS}

We now present our results in this section. An illustrative example with a simple kdV equation can be found in [6]. The infinitesimal transformations of the Lie group with parameter $\epsilon$ are

$$
\bar{t}=t+\xi^{t}(t, x, u, v) \epsilon, \quad \bar{x}=x+\xi^{x}(t, x, u, v) \epsilon, \quad \bar{u}=u+\eta^{u}(t, x, u, v) \epsilon, \quad \bar{v}=v+\eta^{v}(t, x, u, v) \epsilon .
$$

The vector field

$$
x=\xi^{t}(t, x, u, v) \frac{\partial}{\partial t}+\xi^{x}(t, x, u, v) \frac{\partial}{\partial x}+\eta^{u}(t, x, u, v) \frac{\partial}{\partial u}+\eta^{v}(t, x, u, v) \frac{\partial}{\partial v}
$$

is a Lie point symmetry of (3) if

$$
\left.X^{[3]} \Delta_{1}\right|_{\Delta_{1}=0, \Delta_{2}=0}=0,\left.\quad X^{[3]} \Delta_{2}\right|_{\Delta_{1}=0, \Delta_{2}=0}=0 .
$$

Expanding (45) and and splitting on derivatives of $v$ and $u$, we have an overdetermined system of ten PDEs, namely,

$$
\begin{array}{r}
\xi_{u}^{t}=0, \quad \xi_{v}^{t}=0, \quad \xi_{x}^{t}=0, \quad \xi_{u}^{x}=0, \quad \xi_{v}^{x}=0, \quad \xi_{t t}^{t}=0, \quad \xi_{t t}^{x}=0, \quad 3 \xi_{x}^{x}-\xi_{t}^{t}=0, \\
3 \eta^{v}+2 \xi_{t}^{t} v=0, \quad 3 \alpha \eta^{u}+2 \alpha \xi_{t}^{t} u-3 \xi_{t}^{x}=0 .
\end{array}
$$

Solving the system (46) yields

$$
\xi^{t}=A_{1}+3 A_{2} t, \quad \xi^{x}=A 2 x+\alpha A_{3} t+A_{4}, \eta^{u}=-2 A_{2} u+A_{3}, \quad \eta^{v}=-2 A_{2} v,
$$

for arbitrary constants $A_{1}, A_{2}, A_{3}, A_{4}$. Hence from (47), the infinitesimal symmetries of the coupled $\mathrm{KdV}$ Equations ( 3 ) is a Lie algebra generated by the vector fields

$$
x_{1}=\frac{\partial}{\partial t}, \quad x_{2}=\frac{\partial}{\partial x}, \quad x_{3}=\alpha t \frac{\partial}{\partial x}+\frac{\partial}{\partial u}, \quad x_{4}=3 t \frac{\partial}{\partial t}+x \frac{\partial}{\partial x}-2 u \frac{\partial}{\partial u}-2 v \frac{\partial}{\partial v}
$$

The set of all infinitesimal symmetries of coupled $\mathrm{KdV}$ equations forms a Lie algebra and yield the following commutation relations in Table 1.

\begin{tabular}{|c|c|c|c|c|}
\hline$\left[X_{i}, X_{j}\right]$ & $X_{1}$ & $X_{2}$ & $X_{3}$ & $X_{4}$ \\
\hline$X_{1}$ & 0 & 0 & $\alpha X_{2}$ & $3 X_{1}$ \\
\hline$X_{2}$ & 0 & 0 & 0 & $X_{2}$ \\
\hline$X_{3}$ & $-\alpha X_{2}$ & 0 & 0 & $-2 X_{3}$ \\
\hline$X_{4}$ & $-3 X_{1}$ & $-X_{2}$ & $2 X_{3}$ & 0 \\
\hline
\end{tabular}

Table 1: A commutator table for the Lie algebra generated by the symmetries of coupled KdV equation. 
The following Lie groups, for $i=1,2,3,4$, are obtained

$$
\begin{aligned}
& T_{\epsilon_{1}}: \bar{t}=t+\epsilon_{1}, \bar{x}=x, \bar{u}=u, \bar{v}=v, \\
& T_{\epsilon_{2}}: \bar{t}=t, \bar{x}=x+\epsilon_{2}, \quad \bar{u}=u, \bar{v}=v, \\
& T_{\epsilon_{3}}: \bar{t}=t, \bar{x}=x+\alpha \epsilon_{3} t, \quad \bar{u}=u+\epsilon_{3}, \bar{v}=v, \\
& T_{\epsilon_{4}}: \bar{t}=t e^{3 \epsilon_{4}}, \bar{x}=x e^{\epsilon_{4}}, \bar{u}=u e^{-2 \epsilon_{4}}, \bar{v}=v e^{-2 \epsilon_{4}} .
\end{aligned}
$$

The symmetries obtained yield the following symmetry reductions.

$$
X_{1}=\frac{\partial}{\partial t}
$$

Solving the characteristic equations

$$
\frac{\mathrm{d} t}{1}=\frac{\mathrm{d} x}{c}=\frac{\mathrm{d} u}{0}=\frac{\mathrm{d} v}{0}
$$

associated to the operator $X_{1}$ gives the invariants

$$
J_{1}=x, \quad J_{2}=u, \quad J_{3}=v
$$

Hence, we have

$$
u=\varphi(x), \quad v=\psi(x)
$$

for arbitrary functions $\varphi$ and $\psi$. Substituting the expressions for $u$ and $v$ given by (56) into the system (3), we get a system of third order ordinary DEs namely,

$$
\alpha\left[\varphi(x) \varphi^{\prime}(x)-\psi(x) \psi^{\prime}(x)\right]+\beta \varphi^{\prime \prime \prime}(x)=0, \quad \alpha(\varphi(x) \psi(x))^{\prime}+\beta \psi^{\prime \prime \prime}(x)=0 .
$$

Integration of the system (57) yields;

$$
\begin{aligned}
\frac{\alpha}{2}\left[\varphi(x)^{2}-\psi(x)^{2}\right]+\beta \varphi^{\prime \prime}(x) & =C_{1} \\
\alpha[\varphi(x) \psi(x)]+\beta \psi^{\prime \prime}(x) & =C_{2},
\end{aligned}
$$

for arbitrary constants $C_{1}$ and $C_{2}$. If we take

$$
C_{1}=C_{2}=0
$$

the system (58)-(59) becomes

$$
\begin{aligned}
\frac{\alpha}{2}\left[\varphi(x)^{2}-\psi(x)^{2}\right]+\beta \varphi^{\prime \prime}(x) & =0 \\
\alpha[\varphi(x) \psi(x)]+\beta \psi^{\prime \prime}(x) & =0 .
\end{aligned}
$$


To find more solutions of the system (61)-(62), we determine its Lie point symmetries. Using the Lie's algorithm for computing point symmetries, we see that the Lie point symmetries of (61)-(62) are

$$
X_{1}^{*}=\frac{\partial}{\partial x}, \quad X_{2}^{*}=x \frac{\partial}{\partial x}-2 \varphi \frac{\partial}{\partial \varphi}-2 \psi \frac{\partial}{\partial \psi} .
$$

Proceeding as above, we see that the symmetry $X_{1}^{*}$ yields the trivial solution

$$
u=0, \quad v=0
$$

The second symmetry $X_{2}^{*}$ has the characteristic equations

$$
\frac{\mathrm{d} x}{x}=\frac{\mathrm{d} \varphi}{-2 \varphi}=\frac{\mathrm{d} \psi}{-2 \psi}
$$

which provides the invariants

$$
J_{1}=x^{2} \varphi, \quad J_{2}=x^{2} \psi
$$

Letting

$$
\varphi=\frac{\lambda}{x^{2}}, \quad \psi=\frac{\mu}{x^{2}},
$$

substituting the values of $\varphi$ and $\psi$ into (61)-(62) and solving the resulting equations yield:

Case one. Taking

$$
\mu=0
$$

gives

$$
\lambda=0
$$

or

$$
\lambda=-\frac{12 \beta}{\alpha} .
$$

When

$$
\lambda=0, \text { and } \mu=0,
$$

we also get the trivial solution (64). One can easily see that if

$$
\lambda=-\frac{12 \beta}{\alpha} \text {, and } \mu=0 \text {, }
$$

then

$$
\varphi=-\frac{12 \beta}{\alpha x^{2}}, \quad \psi=0,
$$

which is a solution of the system (61)-(62). Hence

$$
u_{1}(t, x)=-\frac{12 \beta}{\alpha x^{2}}, \quad v_{1}(t, x)=0,
$$


is a solution of the coupled $\mathrm{KdV}$ system (3).

Case two. Taking

$$
\lambda=-\frac{6 \beta}{\alpha}
$$

gives

$$
\mu= \pm \frac{6 \beta i}{\alpha}
$$

with $i^{2}=-1$. Consequently,

$$
u_{2}(t, x)=-\frac{6 \beta}{\alpha x^{2}}, \quad v_{2}(t, x)=\frac{6 i \beta}{\alpha x^{2}},
$$

and

$$
u_{3}(t, x)=-\frac{6 \beta}{\alpha x^{2}}, \quad v_{3}(t, x)=-\frac{6 i \beta}{\alpha x^{2}},
$$

are solutions of the coupled $\mathrm{KdV}$ system. Hence Lie group analysis has given us three steady-state solutions for the coupled KdV system under the time translation symmetry $X_{1}=\frac{\partial}{\partial t}$.

$$
x_{2}=\frac{\partial}{\partial x} \text {. }
$$

Solving the characteristic equations

$$
\frac{\mathrm{d} t}{0}=\frac{\mathrm{d} x}{1}=\frac{\mathrm{d} u}{0}=\frac{\mathrm{d} v}{0}
$$

associated to $X_{2}$ gives the invariants

$$
J_{1}=t, J_{2}=u \quad J_{3}=v .
$$

Therefore, the group-invariant solution is

$$
u=\phi(t), \quad v=h(t)
$$

for arbitrary functions $h$ and $\phi$. Substitution of the solutions from (82) into (3), we get a system of first order ordinary DEs, namely,

$$
\phi^{\prime}(t)=0, \quad h^{\prime}(t)=0
$$

which is integrated once with respect to $t$ to yield,

$$
\phi(t)=C_{1}, \quad h(t)=C_{2},
$$

for arbitrary constants $C_{1}$ and $C_{2}$. Consequently, the space translation group-invariant solution of the system (3) is

$$
\begin{gathered}
u(t, x)=C_{1}, \quad v(t, x)=C_{2} . \\
x_{3}=\alpha t \frac{\partial}{\partial x}+\frac{\partial}{\partial u} .
\end{gathered}
$$


Solving the characteristic equations

$$
\frac{\mathrm{d} t}{0}=\frac{\mathrm{d} x}{\alpha t}=\frac{\mathrm{d} u}{1}=\frac{\mathrm{d} v}{0}
$$

associated to Galilean boost gives the invariants

$$
J_{1}=t, \quad J_{2}=v, \quad J_{3}=-u+\frac{x}{\alpha t}, \quad t \neq 0 .
$$

Thus the invariant solution of (3) is

$$
u=\frac{x}{\alpha t}-g(t), \quad v=f(t), \quad t \neq 0,
$$

for arbitrary functions $f$ and $g$. Substitution of the values of $u$ and $v$ from (89) into the System (3), we get a nonlinear system of coupled first order ordinary DEs, namely,

$$
t g^{\prime}(t)+g(t)=0, \quad t f^{\prime}(t)+f(t)=0,
$$

whose solutions are

$$
g(t)=\frac{C_{1}}{t} f(t)=\frac{C_{2}}{t}
$$

for arbitrary constants $C_{1}$ and $C_{2}$. Hence the Galilean boost group-invariant solution of the system (3) is

$$
u(t, x)=\frac{x+A}{\alpha t}, \quad v(t, x)=\frac{C_{2}}{t}
$$

where $A=-\alpha C_{1}$ and $t \neq 0$.

\section{The scaling}

$$
X_{4}=3 t \frac{\partial}{\partial t}+x \frac{\partial}{\partial x}-2 u \frac{\partial}{\partial u}-2 v \frac{\partial}{\partial v}
$$

. By solving of the characteristic equations

$$
\frac{\mathrm{d} t}{3 t}=\frac{\mathrm{d} x}{x}=-\frac{\mathrm{d} u}{2 u}=-\frac{\mathrm{d} v}{2 v}
$$

associated to this symmetry, we obtain the invariants

$$
J_{1}=\frac{x^{3}}{t}, \quad J_{2}=u x^{2}, \quad J_{3}=v x^{2}
$$

Generally, the group-invariant solution pair is

$$
u(t, x)=\frac{f(\lambda)}{x^{2}}, \quad v(t, x)=\frac{g(\lambda)}{x^{2}}, \quad \text { where } \quad \lambda=\frac{x^{3}}{t}
$$

and the functions $f$ and $g$ satisfy the system of third order nonlinear coupled ordinary DEs

$$
\begin{gathered}
2 \alpha\left(g^{2}-f^{2}\right)-\lambda^{2} f^{\prime}+3 \alpha \lambda\left(f f^{\prime}-g g^{\prime}\right)+\beta\left(-24 f+24 \lambda f^{\prime}+27 \lambda^{3} f^{\prime \prime \prime}\right)=0 \\
-4 \alpha f g-\lambda^{2} g^{\prime}+3 \alpha \lambda(f g)^{\prime}+\beta\left(-24 g+24 \lambda g^{\prime}+27 \lambda^{3} g^{\prime \prime \prime}\right)=0 \\
X=X_{1}+c X_{2}
\end{gathered}
$$


We consider a symmetry $X$, which is a linear combination of the time and space translations symmetries, that is,

$$
X=\frac{\partial}{\partial t}+c \frac{\partial}{\partial x}
$$

for a constant $c$. The invariants associated to this symmetry $X$ are

$$
J_{1}=x-c t, \quad J_{2}=u, \quad J_{3}=v .
$$

Hence, the invariant solution for the symmetry $X$ is

$$
u=f(x-c t), \quad v=g(x-c t)
$$

for arbitrary functions $f$ and $g$. Substitution of $u$ and $v$ from (102) into the system (3) yields a system of nonlinear third order ordinary DEs, namely

$$
-c f^{\prime}(\xi)+\alpha\left\{f(\xi) f^{\prime}(\xi)-g(\xi) g^{\prime}(\xi)\right\}+\beta f^{\prime \prime \prime}(\xi)=0, \quad-c g^{\prime}(\xi)+\alpha(f(\xi) g(\xi))^{\prime}+\beta g^{\prime \prime \prime}(\xi)=0
$$

which on integrating once with respect to $\xi$ yields

$$
-c f+\frac{1}{2} \alpha\left(f^{2}-g^{2}\right)+\beta f^{\prime \prime}+C_{1}=0, \quad-c g+\alpha f g+\beta g^{\prime \prime}+C_{2}=0,
$$

for arbitrary constants $C_{1}$ and $C_{2}$.

Remark 3.1. If we take the constants $C_{1}=C_{2}=0$, then when the wave velocity $c=0$, we can recover the stationary solutions given in (3).

Remark 3.2. Traveling wave solutions of the system (3) must satisfy the system (104).

Computation of conservation laws for the coupled KdV Equations (3) is done using two methods; the method of multipliers and a theorem due to Ibragimov. We seek local conservation law multipliers for the system (3), whose determining equations are

$$
\frac{\delta}{\delta u}\left[\Lambda^{1} \Delta_{1}+\Lambda^{2} \Delta_{2}\right]=0, \quad \frac{\delta}{\delta v}\left[\Lambda^{1} \Delta_{1}+\Lambda^{2} \Delta_{2}\right]=0
$$

where

$$
\begin{aligned}
& \frac{\delta}{\delta u}=\frac{\partial}{\partial u}-D_{t} \frac{\partial}{\partial u_{t}}-D_{x} \frac{\partial}{\partial u_{x}}+D_{x}^{2} \frac{\partial}{\partial u_{x x}}-D_{x}^{3} \frac{\partial}{\partial u_{x x x}}+\ldots, \\
& \frac{\delta}{\delta v}=\frac{\partial}{\partial v}-D_{t} \frac{\partial}{\partial v_{t}}-D_{x} \frac{\partial}{\partial v_{x}}+D_{x}^{2} \frac{\partial}{\partial v_{x x}}-D_{x}^{3} \frac{\partial}{\partial v_{x x x}}+\cdots,
\end{aligned}
$$

are the Euler-Lagrange operators and

$$
\begin{aligned}
& D_{t}=\frac{\partial}{\partial t}+u_{t} \frac{\partial}{\partial u}+v_{t} \frac{\partial}{\partial v}+u_{t x} \frac{\partial}{\partial u_{x}}+v_{t x} \frac{\partial}{\partial v_{x}}+u_{t t} \frac{\partial}{\partial u_{t}}+v_{t t} \frac{\partial}{\partial v_{t}}+\cdots \\
& D_{x}=\frac{\partial}{\partial x}+u_{x} \frac{\partial}{\partial u}+v_{x} \frac{\partial}{\partial v}+u_{x x} \frac{\partial}{\partial u_{x}}+v_{x x} \frac{\partial}{\partial v_{x}}+u_{t x} \frac{\partial}{\partial u_{t}}+v_{t x} \frac{\partial}{\partial v_{t}}+\cdots
\end{aligned}
$$


are total derivatives operators. We look for second order multipliers, that is,

$$
\Lambda^{n}=\Lambda^{n}\left(t, x, u, u_{x}, u_{x x}, v, v_{x}, v_{x x}\right), \quad n=1,2 .
$$

The determining Equations (105) become

$$
\begin{aligned}
& \frac{\delta}{\delta u}\left[\Lambda^{1}\left\{u_{t}+\alpha u u_{x}-\alpha v v_{x}+\beta u_{x x x}\right\}+\Lambda^{2}\left\{v_{t}+\alpha u v_{x}+\alpha v u_{x}+\beta v_{x x x}\right\}\right]=0 \\
& \frac{\delta}{\delta v}\left[\Lambda^{1}\left\{u_{t}+\alpha u u_{x}-\alpha v v_{x}+\beta u_{x x x}\right\}+\Lambda^{2}\left\{v_{t}+\alpha u v_{x}+\alpha v u_{x}+\beta v_{x x x}\right\}\right]=0 .
\end{aligned}
$$

Expanding (111)-(112) and splitting on derivatives of $u$ and $v$ yields an overdetermined system of 22 PDEs, namely

$$
\begin{array}{r}
\Lambda_{x x}^{1}=0, \Lambda_{x x}^{2}=0 \Lambda_{v x}^{1}=0, \quad \Lambda_{v x}^{2}=0, \quad \Lambda_{x v_{x x}}^{1}=0, \quad \Lambda_{x v_{x x}}^{2}=0, \quad \beta \Lambda_{v v}^{1}-\alpha \Lambda_{v_{x x}}^{2}=0, \\
\beta \Lambda_{v v}^{2}+\alpha \Lambda_{v v_{x x}}^{1}=0, \quad \Lambda_{v v_{x x}}^{1}=0, \quad \Lambda_{v v_{x x}}^{2}=0, \quad \Lambda_{v_{x x} v_{x x}}^{1}=0, \quad \Lambda_{v_{x x} v_{x x}}^{2}=0, \quad \Lambda_{u}^{1}+\Lambda_{v}^{2}=0, \\
\Lambda_{t}^{1}+\alpha\left(\Lambda_{x}^{2} v+\Lambda_{x}^{1} u\right)=0, \quad \Lambda_{t}^{2}+\alpha\left(\Lambda_{x}^{2} u-\Lambda_{x}^{1} v\right)=0, \quad \Lambda_{u}^{2}-\Lambda_{v}^{1}=0, \quad \Lambda_{u_{x}}^{1}=0, \quad \Lambda_{u_{x}}^{2}=0, \\
\Lambda_{u_{x x}}^{1}+\Lambda_{v_{x x}}^{2}=0, \quad \Lambda_{u_{x x}}^{2}-\Lambda_{v_{x x}}^{1}=0, \quad \Lambda_{v_{x}}^{2}=0 \quad \Lambda_{v_{x}}^{1}=0 .
\end{array}
$$

Calculations reveal the solution of the system (113) as

$$
\begin{aligned}
& \Lambda^{1}=\frac{\alpha}{2 \beta}\left(c_{3}\left\{u^{2}-v^{2}\right\}+2 c_{4} u v\right)+\left(c_{2} t+c_{5}\right) u+\left(c_{1} t+c_{6}\right) v+c_{3} u_{x x}+c_{4} v_{x x}+c_{7}-\frac{1}{\alpha} c_{2} x, \\
& \Lambda^{2}=\frac{\alpha}{2 \beta}\left(c_{4}\left\{u^{2}-v^{2}\right\}-2 c_{3} u v+\right)+\left(c_{1} t+c_{6}\right) u-\left(c_{2} t+c_{5}\right) v+c_{4} u_{x x}-c_{3} v_{x x}+c_{8}-\frac{1}{\alpha} c_{1} x,
\end{aligned}
$$

for arbitrary constants $c_{1}, \ldots, c_{8}$.

Remark 3.3. Essentially, the nonlinear coupled system of KdV Equations (3) has eight sets of local conservation law multipliers.

Solving (105)" we obtain conserved vectors corresponding to each set of multipliers as shown below.

(i) The multiplier

$$
\left(\Lambda_{1}^{1}, \Lambda_{1}^{2}\right)=\left(t v, t u-\frac{x}{\alpha}\right)
$$

has the conserved vectors

$$
T_{1}^{t}=t u v-\frac{x v}{\alpha}, \quad T_{1}^{x}=\beta\left[t\left\{v u_{x x}+u v_{x x}-v_{x} u_{x}\right\}+\frac{1}{\alpha}\left\{v_{x}-x v_{x x}\right\}\right]+\alpha\left[t\left(u^{2} v-\frac{v^{3}}{3}\right)\right]
$$

(ii) The multiplier

$$
\left(\wedge_{2}^{1}, \Lambda_{2}^{2}\right)=\left(t u-\frac{x}{\alpha},-t v\right)
$$


has the conserved vectors

$$
\begin{array}{r}
T_{2}^{t}=\frac{t}{2}\left\{u^{2}-v^{2}\right\}-\frac{x u}{\alpha}, T_{2}^{x}=\beta\left[t\left(u u_{x x}-v v_{x x}+\frac{1}{2}\left\{v_{x}^{2}-u_{x}^{2}\right\}\right)+\frac{1}{\alpha}\left\{u_{x}-x u_{x x}\right\}\right]+ \\
\alpha t\left[\frac{u^{3}}{3}-u v^{2}\right]+\frac{x}{2}\left\{v^{2}-u^{2}\right\} .
\end{array}
$$

(iii) The multiplier

$$
\left(\wedge_{3}^{1}, \Lambda_{3}^{2}\right)=\left(\frac{\alpha}{2 \beta}\left\{u^{2}-v^{2}\right\}+u_{x x},-\left\{\frac{\alpha u v}{\beta}+v_{x x}\right\}\right),
$$

has the conserved vectors

$$
\begin{array}{r}
T_{3}^{t}=\frac{\alpha}{2 \beta}\left(\frac{u^{3}}{3}-u v^{2}\right), \quad T_{3}^{x}=\frac{\alpha}{2}\left[\left(u^{2}-v^{2}\right) u_{x x}-v^{2} v_{x x}\right]-\alpha u v v_{x x}+ \\
\frac{\beta}{2}\left[u_{x x}^{2}-v_{x x}^{2}\right]+u_{t} u_{x}-v_{t} v_{x}+\frac{\alpha^{2}}{4 \beta}\left[\frac{1}{2}\left\{u^{4}+v^{4}\right\}-3 u^{2} v^{2}\right] .
\end{array}
$$

(iv) The multiplier

$$
\left(\wedge_{4}^{1}, \wedge_{4}^{2}\right)=\left(\left\{\frac{\alpha u v}{\beta}+v_{x x}\right\}, \frac{\alpha\left[u^{2}-v^{2}\right]}{2 \beta}+u_{x x}\right),
$$

has the conserved vectors

$$
\begin{aligned}
& T_{4}^{t}=\frac{\alpha}{2 \beta}\left(u^{2} v-\frac{v^{3}}{3}\right), \\
& T_{4}^{x}=\frac{\alpha^{2}}{2 \beta}\left[\left(u^{3} v-u v^{3}\right)\right]+v_{t} u_{x}+u_{t} v_{x}+\frac{\alpha}{2}\left(u^{2}-v^{2}\right) v_{x x}+\left\{\alpha u v+\beta v_{x x}\right\} u_{x x} .
\end{aligned}
$$

(v) The multiplier

$$
\left(\wedge_{5}^{1}, \Lambda_{5}^{2}\right)=(u,-v)
$$

has the conserved vectors

$$
T_{5}^{t}=\frac{1}{2}\left\{u^{2}-v^{2}\right\}, \quad T_{5}^{x}=\beta\left(u u_{x x}-v v_{x x}+\frac{v_{x}^{2}-u_{x}^{2}}{2}\right)+\alpha\left(\frac{u^{3}}{3}-u v^{2}\right) .
$$

(vi) The multiplier

$$
\left(\wedge_{6}^{1}, \Lambda_{6}^{2}\right)=(v, u)
$$

has the conserved vectors

$$
T_{6}^{t}=u v, \quad T_{6}^{x}=\beta\left(v u_{x x}+u v_{x x}-u_{x} v_{x}\right)+\alpha\left(u^{2} v-\frac{v^{3}}{3}\right) .
$$

(vii) The multiplier

$$
\left(\wedge_{7}^{1}, \wedge_{7}^{2}\right)=(1,0)
$$

has the conserved vectors

$$
T_{7}^{t}=u, \quad T_{7}^{x}=\frac{\alpha}{2}\left\{u^{2}-v^{2}\right\}+\beta u_{x x} .
$$


(viii) The multiplier has

$$
\left(\wedge_{8}^{1}, \wedge_{8}^{2}\right)=(0,1)
$$

the conserved vectors

$$
T_{8}^{t}=v, \quad T_{8}^{x}=\alpha u v+\beta v_{x x}
$$

Remark 3.4. It can be verified that

$$
D_{t} T_{i}^{t}+\left.D_{x} T_{i}^{x}\right|_{\Delta_{1}=0, \Delta_{2}=0}=0,
$$

for $i=1, \ldots, 8$.

Remark 3.5. The expressions in (134) are eight conservation laws for the coupled KdV system (3).

Remark 3.6. The presence of multipliers

$$
\left(\Lambda_{7}^{1}, \Lambda_{7}^{2}\right)=(1,0),\left(\Lambda_{8}^{1}, \Lambda_{8}^{2}\right)=(0,1)
$$

manifest that the coupled $\mathrm{KdV}$ equations are themselves conservation laws.

At this point, we derive conserved vectors for coupled $\mathrm{KdV}$ equations (3) by a new theorem due to Ibragimov. The adjoint equations for the nonlinear system coupled KdV Equations (3) are

$$
\Delta_{1}^{*} \equiv f_{t}+\alpha u f_{x}+\alpha v g_{x}+\beta f_{x x x}=0, \quad \Delta_{2}^{*} g_{t}-\alpha v f_{x}+\alpha u g_{x}+\beta g_{x x x}=0 .
$$

The formal Lagrangian $\mathcal{L}$ for the nonlinear coupled system of the KdV Equations (3) and its adjoint Equations (136) is given by

$$
\mathcal{L}=f\left\{u_{t}+\alpha u u_{x}-\alpha v v_{x}+\beta u_{x x x}\right\}+g\left\{v_{t}+\alpha u v_{x}+\alpha v u_{x}+\beta v_{x x x}\right\}
$$

where $f$ and $g$ are new variables. We shall use the Lie point symmetries of the system (3) ,namely

$$
X_{1}=\partial_{t}, \quad X_{2}=\partial_{x}, \quad X_{3}=\alpha t \partial_{x}+\partial_{u}, \quad X_{4}=3 t \partial_{t}+x \partial_{x}-2 u \partial_{u}-2 v \partial_{v},
$$

to derive conserved vectors corresponding to each symmetry below.

Case (i) The symmetry $X_{1}=\frac{\partial}{\partial t}$, yields Lie characteristic functions given by

$$
W_{1}^{1}=-u_{t}, \quad W_{1}^{2}=-v_{t} .
$$

Hence by Ibragimov's theorem [9], the associated conserved vector is given by

$$
\begin{aligned}
T_{1}^{t}= & \alpha\left[f\left\{u u_{x}-v v_{x}\right\}+g\left\{v u_{x}+u v_{x}\right\}\right]+\beta\left\{f u_{x x x}+g v_{x x x}\right\} \\
T_{1}^{x}= & \alpha\left[f\left\{-u u_{t}+v v_{t}\right\}-g\left\{v u_{t}+u v_{t}\right\}\right] \\
& +\beta\left\{f_{x} u_{t x}+g_{x} v_{t x}-u_{t} f_{x x}-v_{t} g_{x x}-f u_{t x x}-g v_{t x x}\right\}
\end{aligned}
$$


Case (ii) The symmetry $X_{2}=\frac{\partial}{\partial x}$, yields Lie characteristic functions

$$
W_{2}^{1}=-u_{x}, \quad W_{2}^{2}=-v_{x}
$$

Therefore by Ibragimov's theorem [9], the associated conserved vector is

$$
T_{2}^{t}=-u_{x} f-v_{x} g, \quad T_{2}^{x}=f u_{t}+g v_{t}+\beta\left\{-u_{x} f_{x x}-v_{x} g_{x x}+f_{x} u_{x x}+g_{x} v_{x x}\right\}
$$

Case (iii) The symmetry

$$
X_{3}=\alpha t \frac{\partial}{\partial x}+\frac{\partial}{\partial u}
$$

yields Lie characteristic functions given by

$$
W_{3}^{1}=1-\alpha t u_{x}, \quad W_{3}^{2}=-\alpha t v_{x}
$$

Hence by Ibragimov's theorem [9], the associated conserved vector is given by

$$
\begin{array}{r}
T_{3}^{t}=f-\alpha t\left\{u_{x} f+v_{x} g\right\}, \\
T_{3}^{x}=\alpha\left[f u+g v+t\left\{u_{t} f+v_{t} g\right\}+\beta t\left\{\frac{f_{x x}}{\alpha t}-u_{x} f_{x x}-v_{x} g_{x x}+f_{x} u_{x x}+g_{x} v_{x x}\right\}\right] .
\end{array}
$$

Case (iv) The symmetry

$$
X_{4}=3 t \frac{\partial}{\partial t}+x \frac{\partial}{\partial x}-2 u \frac{\partial}{\partial u}-2 v \frac{\partial}{\partial v}
$$

yields the Lie characteristic functions

$$
W_{4}^{1}=-2 u-3 t u_{t}-x u_{x}, \quad W_{4}^{2}=-2 v-3 t v_{t}-x v_{x}
$$

Consequently by Ibragimov's theorem [9], the corresponding conserved vector is given by

$$
\begin{aligned}
T_{4}^{t} & =\alpha\left[3 t\left\{f u u_{x}-f v v_{x}+g u v_{x}+g v u_{x}\right\}\right]+\beta\left[3 t\left\{f u_{x x x}+g v_{x x x}\right\}\right] \\
& -2\{f u+g v\}-x\left\{f u_{x}+g v_{x}\right\}, \\
T_{4}^{x} & =x\left\{f u_{t}+g v_{t}\right\}+\beta\left[3\left(f_{x} u_{x}+g_{x} v_{x}+t\left\{f_{x} u_{t x}+g_{x} v_{t x}\right\}\right)\right] \\
& -\alpha\left[2\left(f\left\{u^{2}-v^{2}\right\}+2 g u v\right)+3 t\left(f\left\{u u_{t}-v v_{t}\right\}+g\left\{v u_{t}+u v_{t}\right\}\right)\right] \\
& -\beta\left[x\left\{u_{x} f_{x x}+v_{x} g_{x x}-f_{x} u_{x x}-g_{x} v_{x x}\right\}+2\left\{u f_{x x}+v g_{x x}\right\}\right] \\
& -\beta\left[3 t\left\{f_{x x} u_{t}+g_{x x} v_{t}+f u_{t x x}+g v_{t x x}\right\}+4\left\{f u_{x x}+g v_{x x}\right\}\right] .
\end{aligned}
$$

Remark 3.7. The appearance of arbitrary functions $f(t, x)$ and $g(t, x)$ in the conserved vectors proves the existence of infinite conservation laws for coupled KdV system obtained by Ibagimov's method. 


\section{Conclusion}

In this paper, Lie group analysis was employed in studying a nonlinear coupled $\mathrm{kdV}$ system. A four-dimensional Lie algebra of symmetries was found for the nonlinear coupled system KdV equations. This was spanned by space and time translations, Galilean boost and scaling symmetries where the scaling symmetry acts on four variables. Associated to each symmetry, we obtained symmetry reductions that gave six nontrivial solutions for the coupled system. All the groupinvariant solutions describe the various states of the system. The obtained solutions can be used as a benchmark against numerical simulations. Lastly, we constructed infinite conservation laws of a nonlinear coupled KdV system by using multipliers and a theorem proposed by Nail Ibragimov.

\section{ACKNOWLEDGEMENT}

The first author acknowledges the financial support of AIMS-South Africa and MasterCard Foundation. The authors are also grateful to the referees for their careful reading of the manuscript and valuable comments.

\section{REFERENCES}

[1] D. J. Arigo, Symmetry analysis of differential equations: an introduction, John Wiley \& Sons, 2015.

[2] G. Bluman, S. Anco, Symmetry and integration methods for differential equations, Springer Science \& Business Media, 2008.

[3] G. W. Bluman, S. Kumei, Symmetries and differential equations, Springer Science \& Business Media, 1989.

[4] Bluman, G. W., Cheviakov, A. F., and Anco, S. C, Applications of symmetry methods to partial differential equations, Springer, 2010.

[5] N. H. Ibragimov, Elementary Lie group analysis and ordinary differential equations, Wiley, 1999.

[6] J. Owuor, M. Khalique, Lie Group Analysis of Nonlinear Partial Differential Equations, Lambert Academic Publishers, 2021

[7] N. H. Ibragimov, CRC handbook of Lie group analysis of differential equations, CRC-Press, 1994.

[8] N. H. Ibragimov, Selected works, ALGA publications, Blekinge Institute of Technology, Selected works, 2009.

[9] N. H. Ibragimov, A new conservation theorem, J. Math. Anal. Appl. 333(2007), 311-328. https://doi.org/10. $1016 / j$.jmaa.2006.10.078.

[10] N. H. Ibragimov, A Practical Course in Differential Equations and Mathematical Modelling: Classical and New Methods. Nonlinear Mathematical Models. Symmetry and Invariance Principles, World Scientific Publishing Company, 2009.

[11] C. M. Khalique, S. A. Abdallah, Coupled Burgers equations governing polydispersive sedimentation; a lie symmetry approach. Results Phys. 16(2020), 76-90. https://doi.org/10.1016/j.rinp. 2020.102967.

[12] R. J. LeVeque, Numerical methods for conservation laws, Springer Verlag, New York, 1992.

[13] S Lie, Vorlesungen Aber Differentialgleichungen mit bekannten infinitesimalen Transformationen. BG Teubner, 1891.

[14] I. Mhlanga, C. Khalique, Travelling wave solutions and conservation laws of the Korteweg-de VriesBurgers Equation with Power Law Nonlinearity. Malays. J. Math. Sci. 11(2017), 1-8.

[15] E. Noether, Invariant variations problem, Nachr. Konig. Gissel. Wissen, Gottingen. Math. Phys. Kl, 6(1918), $235-257$.

[16] P. J. Olver, Applications of Lie groups to differential equations, Springer Science \& Business Media, 1993. 
[17] L. Ovsyannikov, Lectures on the theory of group properties of differential equations, World Scientific Publishing Company, 2013.

[18] H. Pie, Symmetry methods for differential equations: a beginners guide, Cambridge University Pres, Cambridge, 2013.

[19] A. M. Wazwaz, Partial differential equations and solitary waves theory, Springer Science \& Business Media, 2010.

[20] N. Hasibun, L. Abdullah, F. Aini, The Improved GG-Expansion Method to the (3 Dimensional Kadomstev-Petviashvili Equation, Amer. J. Appl. Math. Stat. 1(2013), 64-70.

[21] B. Hong, D. Lu, F. Sun, The extended Jacobi Elliptic Functions expansion method and new exact solutions for the Zakharov equations, World J. Model. Simul. 5(2009), 78-109.

[22] S.M. Ege, E. Misirli, The modified Kudryashov method for solving some fractional-order nonlinear equations, Adv. Difference Equ. 2014 (2014), 135. https://doi .org/10.1186/1687-1847-2014-135. 